\title{
BMJ Open Clinical validation of a public health policy-making platform for hearing loss (EVOTION): protocol for a big data study
}

\author{
Giorgos Dritsakis, ${ }^{1,2}$ Dimitris Kikidis, ${ }^{3}$ Nina Koloutsou, ${ }^{4}$ Louisa Murdin, ${ }^{4}$ \\ Athanasios Bibas, ${ }^{3}$ Katherine Ploumidou, ${ }^{5}$ Ariane Laplante-Lévesque, ${ }^{6}$ \\ Niels Henrik Pontoppidan, ${ }^{6}$ Doris-Eva Bamiou ${ }^{1,2,7}$
}

To cite: Dritsakis G, Kikidis D, Koloutsou N, et al. Clinical validation of a public health policy-making platform for hearing loss (EVOTION): protocol for a big data study. BMJ Open 2018;8:e020978. doi:10.1136/ bmjopen-2017-020978

- Prepublication history for this paper is available online To view these files, please visit the journal online (http://dx.doi org/10.1136/bmjopen-2017020978).

Received 5 December 2017 Accepted 8 December 2017

Check for updates

${ }^{1}$ Ear Institute, University College London, London, UK

${ }^{2}$ Royal National Throat Nose \&

Ear Hospital, London, UK

${ }^{3} 1$ st Department of

Otolaryngology, National and

Kapodistrian University of

Athens, Hippocrateion Hospital,

Athens, Greece

${ }^{4}$ Guy's and St Thomas' NHS

Foundation Trust, London, UK

${ }^{5}$ Athens Medical Center, Athens, Greece

${ }^{6}$ Eriksholm Research Centre,

Elsinore, Denmark

${ }^{7}$ NIHR University College London Hospitals Biomedical Research Centre, London, UK

Correspondence to

Professor Doris-Eva Bamiou;

d.bamiou@ucl.ac.uk

\section{ABSTRACT}

Introduction The holistic management of hearing loss $(\mathrm{HL})$ requires an understanding of factors that predict hearing aid (HA) use and benefit beyond the acoustics of listening environments. Although several predictors have been identified, no study has explored the role of audiological, cognitive, behavioural and physiological data nor has any study collected real-time HA data. This study will collect 'big data', including retrospective HA logging data, prospective clinical data and real-time data via smart HAs, a mobile application and biosensors. The main objective is to enable the validation of the EVOTION platform as a public health policy-making tool for HL.

Methods and analysis This will be a big data international multicentre study consisting of retrospective and prospective data collection. Existing data from approximately $35000 \mathrm{HA}$ users will be extracted from clinical repositories in the UK and Denmark. For the prospective data collection, $1260 \mathrm{HA}$ candidates will be recruited across four clinics in the UK and Greece. Participants will complete a battery of audiological and other assessments (measures of patient-reported HA benefit, mood, cognition, quality of life). Patients will be offered smart HAs and a mobile phone application and a subset will also be given wearable biosensors, to enable the collection of dynamic real-life HA usage data. Big data analytics will be used to detect correlations between contextualised HA usage and effectiveness, and different factors and comorbidities affecting $\mathrm{HL}$, with a view to informing public health decision-making.

Ethics and dissemination Ethical approval was received from the London South East Research Ethics Committee (17/L0/0789), the Hippokrateion Hospital Ethics Committee (1847) and the Athens Medical Center's Ethics Committee (KM140670). Results will be disseminated through national and international events in Greece and the UK, scientific journals, newsletters, magazines and social media. Target audiences include HA users, clinicians, policy-makers and the general public.

Trial registration number NCT03316287; Pre-results.
Strengths and limitations of this study

- This is the first big data study collecting both retrospective, and prospective real-time, hearing aid use and context-related, behavioural and physiological data from hearing aid users.

- The data collected will validate a prototype public health policy-making platform to inform decisions regarding hearing loss management.

- The study will deploy a mobile application on a smartphone issued to the hearing aid users that includes self-administered speech in noise testing, cognitive testing and auditory training, and biosensors.

- There is a risk that not all patients will engage with the equipment used in this study, that is, smart hearing aids, smartphones and biosensors.

\section{INTRODUCTION}

Hearing loss (HL) affects approximately one-third of people over the age of 65 and over $5 \%$ of the world's population. ${ }^{1}$ It is the fifth leading cause of years lived with disability, higher than diabetes and visual impairment. ${ }^{2}$ HL prevalence is on the rise worldwide, primarily due to increased noise exposure and increase in the ageing population, with the number of people with disabling HL (ie, pure tone audiogram $>40 \mathrm{~dB}$ ) worldwide rising from 278 million in 2005 to more than 360 million in $2012 .^{1}$ In the UK, the percentage of people suffering from HL (ie, pure tone audiometry (PTA) $>25 \mathrm{~dB}$ ) is expected to exceed $20 \%$ of the population by $2031 .^{3}$ Addressing the needs of a growing ageing population, including hearing-related needs, requires careful resource allocation in health and social care. ${ }^{2}$ HL has recently been associated with a higher risk of dementia, mental illness and depression and an adverse overall effect on general health. ${ }^{4}$ It also has 
important economic consequences, including work discrimination, reduced productivity, unemployment, early retirement and loss of income. ${ }^{56}$ The treatment of HL also has a significant cost estimated to be $€ 213$ billion per year in the European Union. ${ }^{7}$

Currently, the leading management strategy for the vast majority of patients with HL is the provision of hearing aids (HAs). There is evidence that HAs are effective at improving general health-related quality of life (HRQOL), hearing-specific QOL associated with participation in daily activities and listening ability. ${ }^{8}$ However, HA users still face significant challenges, such as listening in noisy environments, poor sound quality and difficulty to select among predefined programmes and settings. ${ }^{9} 10$ As a result, many adults, especially elderly, do not accustom to their HAs and do not use them. ${ }^{10}$ Moreover, it is unclear whether HAs are equally effective for adults with different degrees of severity of HL. ${ }^{11}$ Ideally, HA fitting should: (1) take into account a range of personal and real-life behavioural, physiological and other audiological factors; (2) adapt to the challenging and changing situations for individual HA users on a continuous basis and (3) be supported by individualised rehabilitation treatments such as auditory training. ${ }^{12}$ However, linking such information to appropriate management strategies requires a better understanding of the role of several factors beyond the acoustic characteristics of the various environments in which HAs are worn. These factors include the activities of the HA user in different environments as well as other physiological, cognitive and medical conditions on HA use and benefit.

Several studies have examined mostly demographic and self-reported factors that predict HA uptake and outcomes. The most robust predictors are self-reported hearing disability, age and degree of HL. ${ }^{13-16}$ HA users tend to overestimate their HA usage, as they report using their HAs more than what is measured by automatic HA logs. ${ }^{17} 18$ It is thus important to use objective data logging of HA use for accurate information. This automatic logging has been used in both adults and children with hearing impairments to explore how often, for how long and in which situations HAs are and are not used, as well as the factors that are associated with higher usage. ${ }^{17} 19$ Laplante-Lévesque et $a l^{18}$ showed that these patterns of HA usage are at least as important in predicting usage as the duration of HA use. A recent retrospective data-logging study found no statistical difference in HA use between patients with mild and moderate HL with implications for the prescription of HAs for milder degrees of HL. ${ }^{20}$ However, to the knowledge of the authors, no study to date has looked at associations between audiological, cognitive, behavioural and physiological data and HA use and benefit, nor has any study collected real-time HA data.

The research project 'EVidence-based management of hearing impairments: public health pOlicy making based on fusing big data analytics and simulaTION' (EVOTION; http://www.h2020evotion.eu) aims to build the evidence base for the formulation of public health policies related to the prevention, early diagnosis, long-term treatment and rehabilitation of HL, as well as to the detection and prevention of cognitive decline and the socioeconomic inclusion of individuals with HL. Its ultimate objective is to enable and support a more holistic management of HL at the population level. ${ }^{21}$ To this end, the EVOTION project is developing public health policy decision models (PHPDMs) using a combination of factors potentially affecting the effectiveness of HL treatments and ways in which they can inform public health policy decisions. ${ }^{22}$ PHPDMs define, analyse and address a wide range of public health issues with the aim to provide different stakeholders (eg, ministries, regulatory bodies, non-governmental organisations) with tools for HL-related policy formulation. In addition to these models, EVOTION is developing a public health policy-making platform (hereafter 'the EVOTION platform') including components enabling the static and real-time feeding of data into a data repository. ${ }^{23}$ The present clinical study, which is conducted as part of the EVOTION project, will collect a large set of heterogeneous data including retrospective HA logging data, prospective clinical data and real-time data via smart HAs, a mobile application with tests and auditory training and biosensors.

The primary objective of the clinical study is to feed these data into the EVOTION data repository to enable the validation of the EVOTION model and platform as a public health policy-making tool.

Secondary objectives are to:

1. identify predictors of HA use and outcome;

2. identify predictors of temporary or permanent threshold shift following noise exposure in HA users;

3. identify predictors of cognitive decline;

4. investigate benefits of self-management of HA settings;

5. investigate benefits of auditory training for HA use and outcome.

\section{METHODS AND ANALYSIS \\ Study design}

This big data study consists of two parts.

Part 1: Retrospective data collection: Existing data (anonymised and in grouped format) will be collected and analysed. These data will include demographics, HL levels, cause and duration of HL, medical history and HA usage data.

Part 2: Prospective data collection: It will take place within the National Health System (NHS) of the UK and Greece, and within the private sector in Greece. However, while in the UK there is a standard HA fitting pathway nationwide, there is no such pathway in Greece. Thus, in the UK HA candidates will attend the routine clinical NHS HA fitting pathway but will be fitted with EVOTION smart HAs instead of the standard NHS HAs. In Greece, a dedicated flow chart will be followed for the HA fitting for the 
study purposes. In both countries, patients will undergo a battery of audiological and other assessments (including measures of patient-reported HA benefit, mood, cognition, QOL; see the Prospective data collection: EVOTION HA fitting pathway section). Study participants will be issued with a mobile phone application in addition to the HA and a subset with wearable biosensors, to enable the collection of dynamic real-life HA usage data in different environmental and situational contexts.

The choice of the prospective and retrospective data to be collected was based on factors identified from the medical literature that are potentially associated with HA usage and benefits. The aim of the clinical study is to collect information that will help profile each patient in sufficient detail to support optimal HA fitting and evidence-based policy-making for HL. See table 1 for a full list of the retrospective and prospective data to be collected and figure 1 for the design of the prospective study (part 2).

\section{Setting and participants \\ Retrospective study}

Retrospective data from approximately $35000 \mathrm{HA}$ users will be extracted from existing clinical repositories held in the UK (University College London Hospital and Guy's and St Thomas' NHS Foundation Trust through the NHS Auditbase) and Denmark (Oticon A/S).

\section{Prospective study}

A total of $1260 \mathrm{HA}$ users will be recruited over 12 months in the UK and Greece across the following clinics:

Royal National Throat, Nose and Ear Hospital, London, UK (380 patients)

Guy's and St Thomas' NHS Foundation Trust, London, UK (380 patients)

Hippokrateion Hospital, University of Athens, Athens, Greece (300 patients)

Athens Medical Center, Athens, Greece (200 patients).

Hereafter, the rest of the 'Methods' section focuses on the prospective study.

\section{Eligibility criteria}

Inclusion criteria

age $\geq 18$ years old;

basic understanding of oral and written English/ Greek to complete the assessments (as judged by the treating audiologist);

mild to severe (ie, an average of $20-95 \mathrm{~dB}$ HL) unilateral or bilateral sensorineural HL. ${ }^{24}$

\section{Exclusion criteria}

dementia indicated by the Montreal Cognitive Assessment (MoCA) score $<23^{25}$;

not willing or able to use their HAs for at least 2 hours daily on average;

not willing or able to use a smartphone.
Recruitment

\section{Participant identification}

In the UK, participants will be recruited through the standard NHS HA fitting pathway. These will be patients referred for a HA fitting to the Hearing Aid Centre of the Royal National Throat, Nose and Ear Hospital or to the Audiology Department of Guy's and St Thomas' NHS Foundation Trust. In Greece, participants will be recruited from the NHS Ear, Nose and Throat outpatient clinics and the specialist Neurotology Clinic of the first Otolaryngology Department at the University of Athens and from the private sector department of Audiology, Otology and Neurotology of Athens Medical Centre.

For the above clinics, recruitment will be conducted through one of the following routes:

- Referral to a HA centre by primary/secondary/ tertiary care professionals with preliminary assessment including PTA (UK only).

- Known HA users who require refit or upgrade of their HAs due to either obsolete technology or a change in audiological status (UK only).

- Self-referral/external referral. These patients will require full assessment at first visit including PTA and they may or may not go on to require HA fitting.

- Patients who have already attended the clinic and have been referred for HA fitting but have not been fitted for various reasons (personal choice, postponed appointment, financial reasons).

\section{Consent}

Potential participants will be primarily identified at the point of referral or booking. A member of the clinical (in cases where EVOTION researchers are not part of the routine clinical team) or research team will contact them prior to their clinical appointment and inform them of the opportunity to participate in the study through a patient information sheet and invitation letter at least 48 hours prior to their appointment. Those willing to participate will be provisionally offered an EVOTION appointment (visit 1). Eligibility will be confirmed at visit 1 after further clinical assessment. If the patient is suitable, they will have the opportunity to discuss the purpose and nature of the study, benefits and risks with a member of the research team and ask questions and will then be asked to complete and sign a consent form.

\section{Prospective data collection: EVOTION HA fitting pathway}

Visit 1: assessment

Individuals who have completed the consent form will be asked to complete their standard clinical assessments along with additional research data collection.

Standard prefit assessments include:

- Medical and audiological history: Short clinical interview regarding clinical, medical and occupational history (eg, marital status, history of noise exposure, comorbidities) based on the relevant fields of the NHS Auditbase platform in the UK or equivalent questions in Greece. 
Table 1 Full list of data to be collected in the EVOTION clinical study

\begin{tabular}{|c|c|c|c|c|c|c|c|}
\hline \multirow[b]{2}{*}{ Generic data type } & \multirow[b]{2}{*}{ Specific data type } & \multicolumn{4}{|c|}{ Means of capture } & \multirow[b]{2}{*}{ Retrospective } & \multirow[b]{2}{*}{ Prospective } \\
\hline & & HA & Sensor & Mobile & Clinic & & \\
\hline \multirow[t]{2}{*}{ HA logging } & Periods of HA usage & $\mathrm{x}$ & & & & $\mathrm{x}$ & $\mathrm{X}$ \\
\hline & Use and adjustment of HA controls & $\mathrm{x}$ & & & & & $\mathrm{x}$ \\
\hline \multirow[t]{3}{*}{$\begin{array}{l}\text { TTS episode } \\
\text { related }\end{array}$} & $\begin{array}{l}\text { Predicted TTS values due to noise exposure } \\
\text { and time required for recovery }\end{array}$ & $\mathrm{x}$ & & $\mathrm{X}$ & & & $\mathrm{x}$ \\
\hline & $\begin{array}{l}\text { Time of occurrence and severity of actual } \\
\text { TTS episodes }\end{array}$ & $\mathrm{x}$ & $\mathrm{x}$ & $\mathrm{X}$ & & $\mathrm{x}$ & $x$ \\
\hline & Self-administered PTA at $4 \mathrm{kHz}$ & & & $\mathrm{X}$ & & & $\mathrm{x}$ \\
\hline \multirow[t]{4}{*}{ Audiological } & PTA in quiet & & & & $\mathrm{x}$ & $x$ & $\mathrm{x}$ \\
\hline & Speech in Noise & & & $\mathrm{x}$ & & & $\mathrm{x}$ \\
\hline & $\begin{array}{l}\text { Reaction time (in Speech in Noise and Digit } \\
\text { Span tests) }\end{array}$ & $x$ & & $\mathrm{x}$ & & $\mathrm{x}$ & $x$ \\
\hline & Satisfaction with HA usage & & & $\mathrm{x}$ & $\mathrm{x}$ & & $\mathrm{X}$ \\
\hline \multirow[t]{3}{*}{ Physiological } & Heart rate & & $\mathrm{X}$ & & & & $\mathrm{x}$ \\
\hline & Respiratory rate & & $\mathrm{X}$ & & & & $\mathrm{x}$ \\
\hline & Pulse & & $x$ & & & & $\mathrm{x}$ \\
\hline \multirow[t]{2}{*}{ Cognitive } & Cognitive assessment & & & & $\mathrm{x}$ & & $\mathrm{x}$ \\
\hline & Digit recall test for auditory working memory & & & $x$ & & & $\mathrm{x}$ \\
\hline \multirow[t]{2}{*}{ Mood and QOL } & Mood and anxiety monitoring & & & & $\mathrm{X}$ & & $\mathrm{x}$ \\
\hline & QOL & & & & $\mathrm{x}$ & & $\mathrm{x}$ \\
\hline \multirow{6}{*}{$\begin{array}{l}\text { Clinical and } \\
\text { medication }\end{array}$} & Diabetes & & & & $\mathrm{x}$ & $x$ & $\mathrm{x}$ \\
\hline & Obesity & & & & $\mathrm{x}$ & $\mathrm{X}$ & $\mathrm{x}$ \\
\hline & Family history of $\mathrm{HL}$ & & & & $x$ & $x$ & $x$ \\
\hline & History of medications & & & & $\mathrm{x}$ & $\mathrm{X}$ & $\mathrm{x}$ \\
\hline & Duration of $\mathrm{HL}$ & & & & $\mathrm{x}$ & $x$ & $\mathrm{x}$ \\
\hline & Cause of $\mathrm{HL}$ & & & & $\mathrm{x}$ & $x$ & $x$ \\
\hline \multirow{4}{*}{$\begin{array}{l}\text { Behavioural and } \\
\text { lifestyle }\end{array}$} & Watching television & $\mathrm{x}$ & & $\mathrm{x}$ & & & $x$ \\
\hline & Participating in conversations and meetings & $\mathrm{X}$ & $\mathrm{X}$ & & & & $\mathrm{X}$ \\
\hline & Rating of HA ease or difficulty to use & & & $\mathrm{x}$ & & & $\mathrm{X}$ \\
\hline & Socioeconomic status & & & & $\mathrm{x}$ & & $\mathrm{X}$ \\
\hline \multirow[t]{5}{*}{ Personal } & Education level & & & & $x$ & $\mathrm{X}$ & $x$ \\
\hline & Presence of significant others & & & & $x$ & $x$ & $x$ \\
\hline & Age & & & & $x$ & $x$ & $x$ \\
\hline & Gender & & & & $\mathrm{x}$ & $\mathrm{X}$ & $\mathrm{x}$ \\
\hline & Personal carer & & & & $x$ & $x$ & $x$ \\
\hline Occupational & $\begin{array}{l}\text { Employment history and current status } \\
\text { (including noise exposure) }\end{array}$ & & & & $x$ & $\mathrm{x}$ & $x$ \\
\hline \multirow[t]{3}{*}{ Environmental } & Location & & $x$ & $\mathrm{x}$ & & & $x$ \\
\hline & Noise type, frequency spectrum and level & $\mathrm{x}$ & & & & & $\mathrm{X}$ \\
\hline & Outdoor activities (eg, conversations) & $x$ & $\mathrm{x}$ & & & & $x$ \\
\hline
\end{tabular}

How these data will be collected (ie, automatically logged on the HA, via the wearable biosensor, the mobile phone application or at the clinic) and whether they will be collected at the retrospective (part 1) or the prospective (part 2) study is specified. Also see the 'Study design', 'Prospective data collection: EVOTION HA fitting pathway' and 'Equipment' sections for details.

HA, hearing aid; HL, hearing loss; PTA, pure tone audiometry; QOL, quality of life; TTS, temporary threshold shift. 


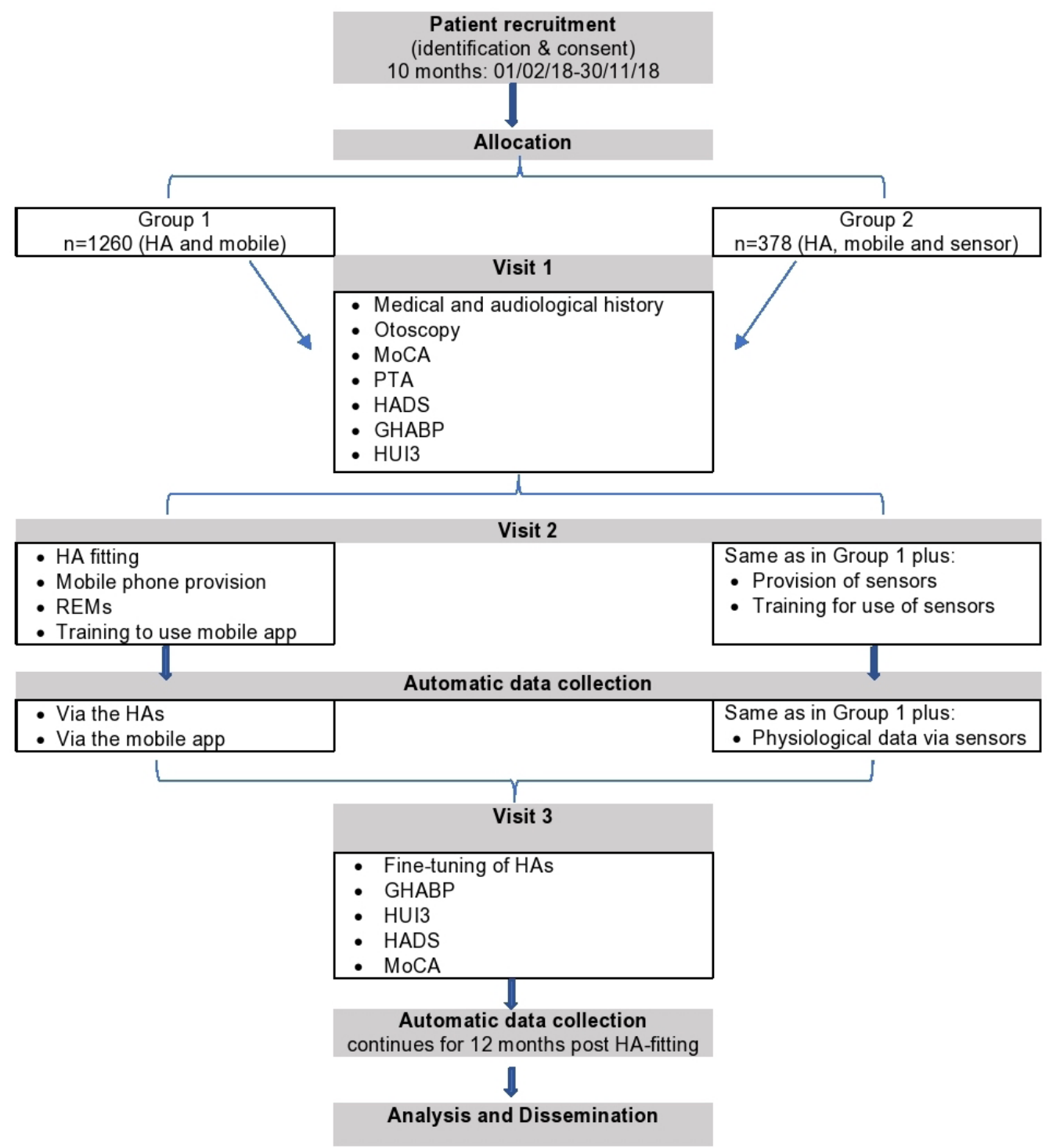

Figure 1 Flow diagram of the prospective data collection (part 2) of the study. See the 'Prospective data collection: EVOTION HA fitting pathway' section for details about the assessments. GHABP, Glasgow Hearing Aid Benefit Profile; HA, hearing aid; HADS, Hospital Anxiety and Depression Scale; HUI3, Health Utility Index Mark 3; MoCA, Montreal Cognitive Assessment; PTA, pure tone audiometry; REM, real-ear measurement.

- Otoscopy, tympanometry, acoustic reflexes.

- PTA if not already available. ${ }^{24}$

- The Glasgow Hearing Aid Benefit Profile (GHABP): Validated self-report instrument assessing listening difficulty, handicap, HA use and benefit across four everyday listening situations. ${ }^{26}$ The GHABP is routinely performed in the UK but not in Greece, where it will be performed as part of the study.

Additional research EVOTION assessments will be:

- The MoCA: Assesses visuospatial/executive function, naming, attention, language, abstraction, memory and orientation to time and place. ${ }^{27} 28$

- The Hospital Anxiety and Depression Scale (HADS): Fourteen-item scale, seven of the items relate to anxiety and the other seven to depression. ${ }^{29} 30$

- The Health Utility Index Mark 3 (HUI3): Health status classification system assessing eight attributes: vision, hearing, speech, ambulation, dexterity, emotion, cognition and pain. ${ }^{31}$ It has demonstrated significant sensitivity to HL. ${ }^{32}$ A license has been obtained from the HUI organisation. It was translated into Greek by the first and second authors for the HUI organisation according to the standard translation process founded on published guidelines for translation and cultural adaptation of HRQOL questionnaires. ${ }^{33}$

Visit 2: HA fitting

After their first visit EVOTION participants will be allocated to an EVOTION HA fitting appointment including:

- Fitting of smart EVOTION HAs using the compression fitting rationale $\mathrm{VAC}+{ }^{34}$ and OpenSound Navigator. ${ }^{35}$ Also please see the 'Equipment' section.

- Verification of the HAs using real-ear measurements. In addition to this, participants will be offered: 
- A mobile phone with an EVOTION application including:

- A self-administered speech-in-noise test: This will be based on the Speech-in-Babble test ${ }^{3637}$ that employs eight lists of monosyllabic phonemically balanced meaningful English words as the speech stimulus presented with multitalker babble as the masker.

- An auditory training programme: This will be based on the Story in Noise, an auditory training programme using words in phrases spoken by adult British female and male talkers. ${ }^{38}$ Phrases are taken from a connected narrative taken from books aimed at foreign learners of English with a background of continuous steady-state speech-shaped noise. The listener is asked to click on one to three keyword(s) present in the target phrase from a set of two to six options, each incorrect answer being phonetically similar to the target. Corrective feedback is given. The phrase is replayed every time a wrong choice is made. Every auditory training session lasts $15 \mathrm{~min}$. Equivalent auditory training material is under development in Greek.

- A digit recall test: This measure of auditory working memory is based on the digit span subtest of the Wechsler Adult Intelligence Scale IV. ${ }^{39}$ Pairs of prerecorded spoken digit sequences will be played and the user will have to type in the sequences in the right or reverse order. On successful recall of at least one sequence from each pair, the sequence increases by one digit (maximum eight digits for forward and seven for backward recall). Discontinuation occurs when both sequences are recalled incorrectly.

- A self-administered PTA: This will measure the pure tone threshold at $4 \mathrm{kHz}$ where temporary threshold shifts are indicative of exposure to too loud sounds. The measurement follows Bekesy audiometry, where the intensity varies continuously and the user indicates whenever they can hear the tone by pressing and holding the on-screen button during the audible phase and releasing the button when they cannot hear the tone. The logged sound environment data then enables the mobile to calculate the actual level of the tone at the EVOTION HAs, and thus calibrate the measurement.

- Other self-reported measures: These will include ratings of HA benefit and self-reporting of noise exposure that could lead to temporary or permanent threshold shift events.

- Biosensors integrated in a commercially available wristband: The biosensors collect physiological data associated with listening effort, that is, heart rate, respiratory rate and pulse. ${ }^{40}$

- Training on how to use the mobile application and biosensors.

For the 12 months following the time of fitting, continuously data will be automatically collected via:
- The EVOTION HAs (audiological, behavioural, cognitive and environmental data; see table 1 for details).

- The EVOTION mobile app (table 1).

- The biosensors (physiological data; table 1).

\section{Visit 3: follow-up}

At approximately 6-8 weeks postfitting, participants will be invited to attend a follow-up appointment including fine-tuning of HAs if required. Participants will also repeat the following assessments as in visit 1: the GHABP, the HUI3, the HADS and the MoCA.

\section{Proposed sample size}

This is a big data study and therefore traditional sample size calculations do not apply. The study will collect data to be fed in a public health policy decision support model, therefore the sample should be as large as possible. A recent big data study by Timmer $e t a l^{20}$ used data from $8500 \mathrm{HA}$ users. In the present study, the total sample size of 1250 patients is the maximum number of patients that can be recruited based on available resources due to the requirements of the study, that is, continuous realworld data collection and number of sources. Given the types of data and time points per patient, the expected data points for this sample exceeds 700 million. Even though the sample size is larger that of traditional clinical trials with individuals with HL, there are no concerns with regards to enrolling a high number of patients in the study as this is an observational study with no substantial harm expected for the participants (also see the 'Data confidentiality' section). The higher number relative to other studies can compensate for the possible variation in real-world data as opposed to lab-based data and for the risk of missing data due to the fact that data collection is partially in the hands of the participants. The high sample size allows broader inclusion criteria and therefore a wider coverage of degrees of HL.

\section{Equipment}

All participants will receive EVOTION HAs and mobile phones. Thirty per cent of participants will receive biosensors, based on their ability and experience to use a wristband and the availability of the devices. EVOTION HAs are manufactured by Oticon A/S. They are a research prototype (EVOTION-12) based on the CE marked and commercially available Oticon Opn. The physical, mechanical and electro-acoustic design of the EVOTION HAs are identical to the Opn HAs. However, the EVOTION HAs enable: (1) logging of HA use, volume changes, intentional and automatic programme shifts and sound environment measures and (2) adjustment to the volume and programmes via a paired Bluetooth connection and the mobile app. EVOTION HAs are suitable for people with mild to severe HL. The mobile phone used in the study is the Samsung Galaxy A3 (2017) and the wearable biosensor will be the Huawei Fit. Participants can keep their mobile phone and biosensor after the end of the 12-month study period but will have to return these if they 
withdraw during the study period. If any of the devices is damaged during the study, support or replacement will be offered. Participants can keep their EVOTION HAs after the end of the 12-month study period. However, if the devices are damaged beyond the end of the 24-month HA warranty period, it will not be possible to repair or replace these and participants in the UK will be offered standard NHS HAs.

\section{Data analysis}

All data will be analysed using big data analytic techniques. ${ }^{42}$ The focus will be on detecting patterns of (1) contextualised HA usage and effectiveness for different types of HL and (2) correlations between different factors and comorbidities affecting HL. Big data analytics will include statistics (eg, descriptive statistics, statistical testing and inference techniques) and data mining (eg, clustering and prediction), as the PHPDMs (see the 'Introduction' section) require. The specification of analytic tasks in these models will define the input data sets, the exact analytic techniques and the form of outputs that the analysis will produce. An electronic record of these specifications will be held in the EVOTION platform and will be linked to the data used in the task, the outputs it produced and the member of the research team who initiated the analysis and will also have access to its outcomes. This will ensure full transparency and auditability of all analytic tasks as well as the controlled and traceable access to the analysis outputs.

\section{Data handling and management}

Data collected at the clinic may be initially entered in a paper or electronic case report form (CRF; only in the UK), in the respective local UK NHS patient system (as per routine care) or directly into a purpose-built electronic database, that will be part of the EVOTION platform. These data will then be transferred to the EVOTION data repository. Where available, hard copies of the CRFs will be held by the clinics who collected them. Data collected via the mobile phones will be automatically transmitted to the data repository. Access to these data will be provided only to approved members of the research team, based on an electronic access control system. Data collected by HAs and sensors will be transmitted to the mobile phone via Bluetooth connection and subsequently to the data repository. An internal Data Monitoring Committee will (1) decide regarding the retention of data in the EVOTION data repository after the end of the project and (2) establish rights to access the anonymised data in line with the UK and Greek research governance frameworks as well as the General Data Protection Regulation.

\section{Data confidentiality}

The member of the research team entering the data will be authenticated before the data entry starts. The system will be monitored for inactivity and will be automatically logged out, if detected to be inactive for a prespecified period of time, to ensure that no unauthorised person can get access to the EVOTION platform and data repository. Data will be transmitted in an anonymised form across all the communication lines between HAs, mobile phones and sensors, and the EVOTION platform itself. Device authentication and data integrity checks will also be in place. The transmission of data from the mobile phones to the EVOTION platform and data repository will be encrypted to ensure the confidentiality and integrity of the transmitted data. The data will be stored in the EVOTION data repository in a fully anonymised form. The identity of the device(s) from which the data were obtained will be pseudoanonymised and will not be subjected to full de-identification as there might be circumstances where, following the analysis of data associated with a particular device, clinicians might need to advise or alert the patients.

\section{Trial organisation and monitoring}

The Chief Investigator of the study (D-EB) will ensure that the EVOTION research team conducts adequate monitoring activities, including adherence to the protocol, compliance with procedures for consenting and reporting adverse events and data quality. She or the principal investigator for each site will inform the sponsor should they have concerns that arise from monitoring or oversight procedures. In addition, and according to the Medical Research Council Guidelines for Good Clinical Practice (1998), a trial management group (TMG) and a trial steering committee (TSC) will also be convened. The TMG will be responsible for the day-to-day management of the trial and, as the study is not a formal clinical trial and is of low risk, will report directly to the TSC. The TSC will review and examine legal, ethical, data protection, privacy and security issues.

\section{Patient and public involvement}

The development of this protocol has been informed by stakeholder consultation with HA users, clinicians, policy-makers and the general public collected in another study within the EVOTION project. The study has also been discussed with and has received input by an external advisory board that was established for the needs of the project including people with HL and other professionals outside the EVOTION research team.

\section{Ethics}

Written informed consent will be taken by research personnel who have received Good Clinical Practice training (in the UK) or relevant education (in Greece). Participants will be entitled to withdraw from the study at any point without giving a reason. Data obtained up to the point of withdrawal will be analysed unless the participant requests it is withdrawn. This will not in any way affect their standard clinical care. In the case of withdrawal participants will be asked to return the equipment received during the study. Also see the 'Equipment' section. 


\section{Dissemination}

The study will be presented in national and international events and conferences in Greece and the UK, will be published in scientific journals and will also be disseminated through newsletter articles, magazines and social media. Target audiences will include HA users, clinicians, policy-makers and the general public.

\section{Twitter \#h2020evotion}

Acknowledgements We would like to thank the following people for their contribution at various stages of the preparation of this clinical protocol: Professor George Spanoudakis (City University, London, UK), Panagiotis Katrakazas (National Technical University of Athens, Athens, Greece), Professor George Gavalas and Mr Apostolos Economou (Athens Medical Center), Professor Marco Cremonini (University of Milan) and Professor Anne Schilder and the evidENT team (UCL Ear Institute and Royal National Throat Nose \& Ear Hospital, London, UK).

Contributors DK, D-EB, NK, LM, AB, NHP and GD made substantial contributions to the conception and design of the work throughout the grant preparation or ethics application process and approved the final manuscript. GD and D-EB led the work and took overall responsibility of the manuscript. GD, D-EB, NK and LM were responsible for the parts of the protocol specific to the UK study. DK, $A B$ and KP were responsible for the parts specific to the Greek study. NHP and AL-L were responsible for the parts of the protocol specific to the HAs.

Funding This project has received funding fromthe European Commission's Horizon 2020 research and innovation programme under grant agreement no. 727521.

Competing interests Oticon A/S, which manufactures the HAs used in this study, is an EVOTION project partner but not the sponsor. The EVOTION HA is a researchonly extension based on the 0ticon Opn HA made specifically for this project.

Patient consent Not required.

Ethics approval Ethical approval was received from the London South East Research Ethics Committee (17/L0/0789), the Hippokrateion Hospital Ethics Committee (1847) and the Athens Medical Center Ethics Committee (KM140670).

Provenance and peer review Not commissioned; peer reviewed for ethical and funding approval prior to submission.

Open Access This is an Open Access article distributed in accordance with the Creative Commons Attribution Non Commercial (CC BY-NC 4.0) license, which permits others to distribute, remix, adapt, build upon this work non-commercially, and license their derivative works on different terms, provided the original work is properly cited and the use is non-commercial. See: http://creativecommons.org/ licenses/by-nc/4.0/

(C) Article author(s) (or their employer(s) unless otherwise stated in the text of the article) 2018. All rights reserved. No commercial use is permitted unless otherwise expressly granted.

\section{REFERENCES}

1. World Health Organisation. Deafness and hearing loss. 2017.

2. Vos T, Barber RM, Bell B, et al. Global, regional, and national incidence, prevalence, and years lived with disability for 301 acute and chronic diseases and injuries in 188 countries, 1990-2013: a systematic analysis for the Global Burden of Disease Study 2013. Lancet 2015;386:743-800.

3. Hill S, Holton K, Regan C. Action Plan on Hearing Loss [Internet]. NHS England, Department of Health Guidance, 2015. https://www. england.nhs.uk/wp-content/uploads/2015/03/act-plan-hearing-lossupd.pdf

4. Livingston G, Sommerlad A, Orgeta V, et al. Dementia prevention, intervention, and care. Lancet 2017;6736.

5. World Health Organisation. Global costs of unaddressed hearing loss and cost-effectiveness of interventions: a WHO report. 2017:1-39.

6. Shield B. Evaluation of the social and economic costs of hearing impairment. Hear-it 2006;202.

7. Kaplan W, Wirtz V, Mantel A, et al. Priority Medicines for Europe and the World Update 2013 report. Methodology 2013;2:7. http://www. who.int/entity/medicines/areas/policy/12-Laing.pdf
8. Ferguson MA, Kitterick PT, Chong LY, et al. Hearing aids for mild to moderate hearing loss in adults. Cochrane Database Syst Rev 2017;9:CD012023.

9. Dillon H. Hearing Aids. 2nd edn. New York: Thieme; 2012.

10. McCormack $A$, Fortnum $\mathrm{H}$. Why do people fitted with hearing aids not wear them? Int J Audiol 2013;52:360-8. http://www.tandfonline. com/doi/full/

11. Timmer BH, Hickson L, Launer S. Adults with mild hearing impairment: are we meeting the challenge? Int J Audiol 2015;54:786-95. http://www.tandfonline.com/doi/full/

12. Ferguson MA, Henshaw $\mathrm{H}$. Auditory training can improve working memory, attention, and communication in adverse conditions for adults with hearing loss. Front Psychol 2015;6:1-7.

13. Gopinath B, Schneider J, Hartley D, et al. Incidence and predictors of hearing aid use and ownership among older adults with hearing loss. Ann Epidemiol 2011;21:497-506.

14. Laplante-Lévesque A, Hickson L, Worrall L. What makes adults with hearing impairment take up hearing AIDS or communication programs and achieve successful outcomes? Ear Hear 2012;33:79-93.

15. Meyer $C$, Hickson L. What factors influence help-seeking for hearing impairment and hearing aid adoption in older adults? Int $J$ Audiol 2012;51:66-74. http://www.tandfonline.com/doi/full/

16. Knudsen LV, Oberg M, Nielsen C, et al. Factors influencing help seeking, hearing aid uptake, hearing aid use and satisfaction with hearing aids: a review of the literature. Trends Amplif 2010;14:127-54. http://journals.sagepub.com/doi/

17. Walker EA, Spratford M, Moeller MP, et al. Predictors of hearing aid use time in children with mild-to-severe hearing loss. Lang Speech Hear Serv Sch 2013;44:73-88.

18. Laplante-Lévesque A, Nielsen C, Jensen LD, et al. Patterns of hearing aid usage predict hearing aid use amount (data logged and self-reported) and overreport. J Am Acad Audiol 2014;25:187-98. http://openurl.ingenta.com/content/xref?genre=article\&issn=10500545 \& volume $=25 \&$ issue $=2 \&$ spage $=187$

19. Muñoz K, Preston E, Hicken S. Pediatric hearing aid use: how can audiologists support parents to increase consistency? J Am Acad Audiol 2014;25:380-7. http://openurl.ingenta.com/content/xref? genre $=$ article\&issn $=1050-0545 \&$ volume $=25 \&$ issue $=4 \&$ spage $=380$

20. Timmer BHB, Hickson L, Launer S. Hearing aid use and mild hearing impairment: Learnings from big data. J Am Acad Audiol 2017;28:731-41.

21. Spanoudakis G, Kikidis D, Bibas A, et al. Public health policy for management of hearing impairments based on big data analytics: EVOTION at Genesis. Washington, DC USA, 2017.

22. Katrakazas P, Trenkova L, Milas J, et al. The EVOTION Decision Support System: Utilizing It for Public Health Policy-Making in Hearing Loss. Stud Health Technol Inform 2017;238:88-91.

23. Prasinos M, Spanoudakis G, Koutsouris D, 29th International Conference on Software Engineering \& Knowledge Engineering, 5-7 Jul 2017. Towards a Model-Driven Platform for Evidence based Public Health Policy Making. Pittsburgh USA, 2017.

24. BSA. Recommended procedure: Pure-tone air-conduction and boneconduction threshold audiometry with and without masking. 2011.

25. Larner AJ. Screening utility of the Montreal Cognitive Assessment (MoCA): in place of - or as well as - the MMSE? Int Psychogeriatr 2012;24:391-6. http://www.journals.cambridge.org/abstract S1041610211001839

26. Gatehouse S. Glasgow Hearing Aid Benefit Profile: derivation and validation of a client-centered outcome measure for hearing aid services. J Am Acad Audiol 1999;10:80-103.

27. Nasreddine ZS, Phillips NA, Bédirian V, et al. The Montreal Cognitive Assessment, MoCA: a brief screening tool for mild cognitive impairment. J Am Geriatr Soc 2005;53:695-9. http://onlinelibrary. wiley.com/doi/

28. Konstantopoulos K, Vogazianos P, Doskas T. Normative Data of the Montreal Cognitive Assessment in the Greek Population and Parkinsonian Dementia. Arch Clin Neuropsychol 2016;31:246-53.

29. Zigmond AS, Snaith RP. The hospital anxiety and depression scale. Acta Psychiatr Scand 1983;67:361-70.

30. Michopoulos I, Douzenis A, Kalkavoura C, et al. Hospital Anxiety and Depression Scale (HADS): validation in a Greek general hospital sample. Ann Gen Psychiatry 2008;7:4. http://annals-generalpsychiatry.biomedcentral.com/articles/

31. Horsman J, Furlong W, Feeny D, et al. The Health Utilities Index (HUI): concepts, measurement properties and applications. Health Qual Life Outcomes 2003;1:54. http://hqlo.biomedcentral.com/ articles/

32. Grutters JP, Joore MA, van der Horst F, et al. Choosing between measures: comparison of EQ-5D, HUI2 and HUI3 in persons with hearing complaints. Qual Life Res 2007;16:1439-49. 
33. Guillemin F, Bombardier C, Beaton D. Cross-cultural adaptation of health-related quality of life measures: literature review and proposed guidelines. J Clin Epidemiol 1993;46:1417-32.

34. Le Goff N, Schum DJ. Evidence on Soft Speech Booster. 2015

35. Le Goff N, Jensen J, Pedersen MS, et al. An introduction to OpenSound Navigator ${ }^{\mathrm{TM}}$. Oticon A/S, white Pap [Internet] 2016:1-9. https://www.oticon.com/ /media/Oticon US/main/ Download Center/White Papers/15555-9950 - OpnSound Navigator.pdf

36. Spyridakou C, Luxon LM, Bamiou DE. Patient-reported speech in noise difficulties and hyperacusis symptoms and correlation with test results. Laryngoscope 2012;122:1609-14.

37. Bamiou DE, lliadou VV, Zanchetta S, et al. What Can We Learn about Auditory Processing from Adult Hearing Questionnaires? J Am Acad Audiol 2015;26:824-37. http://www.scopus.com/inward/record.url? eid=2-s2.0-84946826048\&partnerID=tZOtx3y1
38. Loo JH, Rosen S, Bamiou DE. Auditory Training Effects on the Listening Skills of Children With Auditory Processing Disorder. Ear Hear 2016;37:38-47.

39. Wechsler D. Wechsler adult intelligence scale. 3rd edn. San Antonio, Texas: The Phsychological Corporation, 1997.

40. Mackersie CL, MacPhee IX, Heldt EW. Effects of hearing loss on heart rate variability and skin conductance measured during sentence recognition in noise. Ear Hear 2015;36:145-54. http:// content. wkhealth.com/linkback/openurl?sid=WKPTLP:landingpage\& an $=00003446-201501000-00015$

41. Tietz LHB, Katrakazas P, Laplante-Lévesque A, et al. Associations Between Hearing Performance and Physiological Measures - An Overview and Outlook. Stud Health Technol Inform 2017;238:100-3.

42. Fang R, Pouyanfar S, Yang Y, et al. Computational Health Informatics in the Big Data Age. ACM Comput Surv 2016;49:1-36. http://dl.acm. org/citation.cfm?doid=2911992.2932707 